\title{
Effectiveness, Uses and Safety of Granulocyte Colony Stimulating Factor biosimilars: a protocol for systematic review and meta-analysis
}

Chi-kadibia Theophilus Ukoma

Federal Medical Centre Keffi, Nasarawa, Nigeria

Emmanuel Okechukwu Nna ( $\sim$ e.nna@themping.org)

The Molecular Pathology Institute, Enugu https://orcid.org/0000-0001-6791-2336

Helen Chioma Okoye

UNTH: University of Nigeria Teaching Hospital

Augustine Nwakuche Duru

UNTH: University of Nigeria Teaching Hospital

Samuel Osobuchi Ngene

University of Ibadan College of Medicine

Amaka Obiageli Nnamani

UNTH: University of Nigeria Teaching Hospital

Richard Chinaza Ikeagwulonu

Alex Ekwueme Federal University Teaching Hospital Abakaliki

Chinonyelum Thecla Ezeonu

Alex Ekwueme Federal University Teaching Hospital Abakaliki

Jonathan C Daboer

University of Jos, Department of Community Medicine

Uzoma Vivian Asiegbu

Alex Ekwueme Federal University Teaching Hospital Abakaliki

Chioma Laura Odimegwu

UNTH: University of Nigeria Teaching Hospital

Ifeoma Joy Okoye

UNTH: University of Nigeria Teaching Hospital

Protocol

Keywords: G-CSF, Biosimilar, Neupogen, Febrile Neutropenia, Neutropenic fever, Peripheral Stem Cells

Posted Date: February 3rd, 2021

DOI: https://doi.org/10.21203/rs.3.rs-195467/v1

License: @ (1) This work is licensed under a Creative Commons Attribution 4.0 International License. Read Full License 


\section{Abstract}

Background Granulocyte Colony-stimulating factors (G-CSF) biosimilars are recombinant biologics that are similar to a reference product, neupogen, a 175 amino acid recombinant human G-CSF. Characteristically, biosimilars are produced from living cells as high molecular weight, heterogenous compounds that are highly immunogenic. They are primarily used to treat febrile and severe neutropenia in oncology patients as well as mobilize peripheral stem cells in transplant donors. However, as biosimilars are produced by biological processes rather than chemical synthesis, their comparable effectiveness and safety are very paramount to clinical uses. We aimed to produce a protocol for consistent and accurate systematic review and meta-analysis of G-CSF biosimilars.

Methods We developed a search strategy using MeSH terms, key words and entry terms to search 9 databases: PubMed, AJOL, Embase, Google Scholar, Scopus, Cochrane Library, CINAHL, Web of Science and ResearchGate. Only randomized controlled trials retreivable in the English language will be included in this study. The primary measurable outcomes in this study are uses, effectiveness and safety of G-CSF biosimilars. Identified primary studies will be screened, deduplicated and selected based on study design, inclusion/exclusion criteria and outcome measures using DistillerSR software. Studies will be assessed for methodological, clinical and statistical heterogeneity. Extractable data items for effectiveness measure are: i) proportion of patients with $50 \%$ increase in absolute neutrophil count within 3-7 days; ii) resolution of fever within 3-7 days, iii) resolution of intra-oral mucosa ulcers within 7-10 days and iv) resoluton of difficulty in swallowing within 7-10 days. Measures of safety are i) proportion of patients with immunologic reactions, ii) any other documented adverse events. Quality scores and risk of bias for individual studies will be reported. Funnel Plots will be used for assessing publication bias in selected studies. Effect size, variance, SE and \% $\mathrm{Cl}$ and heterogeneity tests will be reported on forest plots using the CMA software version 3. Subgroup analysis and meta-regression will also be included using secondary outcomes as moderators and explanatory variables. The systematic review and meta-analysis will be reported according to PRISMA 2015 Statement.

Discussion Ethical approval will not be required since this study will be based on published data. G-CSF biosimilars will be compared with the reference product, neupogen (filgrastin). The uses, effectiveness and safety of the biosimilars will be discussed. The study will also examine short and long acting biosimilars and compare their overall effectiveness. The strength of evidence from this study will be assessed using the NIH Quality assessment for systematic review and meta-analysis.

Trial Registration Number The study is registered with PROSPERO, with registration number CRD42021232375

\section{Background}

The uses and effectiveness of Granulocyte Colony-stimulating factors (G-CSF) in the management of haematological malignancies, solid tumours and mobilization of stem cells from peripheral blood have long been established [1-3]. Both the American Society of Clinical Oncology (ASCO) and the European Organization for Research and Treatment of Cancer (EORTC) guidelines recommend the use of G-CSF for the treatment and prevention of febrile neutropenia (FN) in haematologic malignancy and other oncology cases in patients undergoing chemotherapy $[4,5]$.

G-CSF is the primary regulator of proliferation and differentiation, maturation, survival, and functions of granulocytes to exert the biological defense mechanism $[6,7]$. The discovery of filgastrim $[8]$ and the approval of its biosimilars $[9,10]$ have revolutionized cancer treatment leading to increased survival of patients undergoing chemotherapy.

G-CSFs are a type of haemopoietic growth factors which are synthesized mainly in the bone marrow to aid stimulation, proliferation, differentiation, and survival of neutrophil precursors in the bone marrow and to promote their maturation process. Its use gain popularity among cancer patients who had chemotherapy- or radiotherapy-induced neutropenia where it reduced the degree and duration of neutropenia [7,10]. It has been used for other causes of severe neutropenia. It is also used in patients undergoing stem cell transplantation to mobilize peripheral stem cells. G-CSF exerts its effect on its target by binding to and forming complexes with the haematopoietin receptor family. Once bound to the receptors, they form G-CSF-Receptor ligand complex which causes dimerization of the receptor and signal transduction through proteins including Jak, Lyn, STAT and Erk1/2 [11].

In 2016, G-CSF and other biologics contributed significantly to global expenditure on medicare, causing increase in healthcare spending [12,13]. Biosimilars are biologics with high similarity but not identical to a reference drug. They are believed to offer some reduction in global cost of drugs with a potential of saving up to $22 \%$ of the cost on the biologics over a 10 -year period in the United States $[14,15]$. Apart from reduction in cost, biosimilars have become readily available and accessible to many patients [13]. G-CSF biosimilars are already in use and they show similar effects with G-CSF [16]. Generally, biosimilars are usually subjected to structural, biochemical and clinical comparative analysis to the reference products by regulatory bodies [17-20].

G-CSF biosimilars are recombinant biologics that are similar to a reference product, neupogen, a 175 amino acid recombinant human G-CSF [21]. They are classified into a) shorting acting such as the lenograstim, filgrastim which includes neupogen, zarzio, nivestim and accofil; and b) the long-acting G-CSFs such as the pegylated filgrastim and lipegfilgrastim. Presently, European Medicines Agency has approved about twenty-one G-CSF biosimilars for clinical use [22] while the FDA approved pegfilgrastim (Neulasta) in 2002 as a long-acting form of G-CSF [23]. FDA also approved three biosimilars: pegfilgrastim-jmdb (Fulphila), pegfilgrastim-cbqv (Udenyca; Coherus BioSciences) [24] and pegfilgrastim-apgf (Nyvepria) [25]. Characteristically, biosimilars are produced from living cells as high molecular weight, heterogeneous compounds that are highly immunogenic [26]. Although they are difficult to fully characterize, their efficacy, safety and uses are similar. Their supply is highly dependent on stringent manufacturing processes that are very exclusive to each product. Overall, biosimilars have reduced cost of medicare for cancer patients. Fundamentally, biosimilars are different from generics, a term used mainly for off-patent chemically synthesized drugs that are similar to a parent drug. Generics have a well-defined chemical structure; they are small molecules with identical copies in the manufacturing process [27]. They are mostly non-immunogenic and very stable to external variations. With increasing approvals of G-CSF biosimilar medicines, by both FDA and EMA, some new and promising biosimilars may still not receive approval on reasons of lack of optimal effectiveness and safety. 
Generally, G-CSF biosimilars are indicated for neutropenia and/or peripheral stem cell mobilization. We aim to develop a protocol to enable a reliable, robust and accurate systematic review and meta-analysis of the uses, effectiveness and safety of G-CSF biosimilars.

\section{Methods}

\section{OBJECTIVE}

The overall objective of this study is to evaluate the effectiveness, uses and safety of G-CSF biosimilars in oncology patients undergoing chemotherapy, nononcology patients with severe neutropenia and peripheral mobilization of stem cells.

The study will determine the effects and impacts of long-acting G-CSF compared to that of short acting forms in reducing neutropenic fever in oncology patients undergoing chemotherapy.

\section{Study objectives:}

1. To determine the overall effectiveness of G-CSF biosimilars.

2. To assess the indications for G-CSF biosimilars.

3. To catalogue clinically available G-CSF biosimilars and factors affecting their uses.

4. To compare the differences in the effectiveness of short vs long-acting G-CSF biosimilars.

5. To evaluate the safety profile of G-CSF biosimilars.

\section{Review Questions:}

Specific questions to be answered by this review include:

1. How effective are G-CSF biosimilars in managing and preventing neutropenia?

2. How effective are G-CSF biosimilars in peripheral stem cell mobilization in transplant medicine?

3. Does G-CSF biosimilars improve clinical outcome in cancer patients undergoing chemotherapy?

4. What are the types of G-CSF biosimilars approved and available in the market?

5. What are the identified adverse effects of G-CSF biosimilars?

6. What is the rationale for using either short or long-acting G-CSF biosimliars?

\section{Study Characteristics}

a. Study Design: This is a protocol designed for a consistent and accurate systematic review and meta-analysis of the effectiveness, uses and safety of G-CSF biosimilars. It is focussed on interventional studies including randomized clinical trials and quasi-clinical trials. There is no time restriction for eligible studies.

b. Inclusion Criteria: The specific inclusion criteria for selecting studies are:

i. Interventional studies: only RCTs and quasi-clinical trials in humans will be included.

ii. Studies are retrievable in the English language.

iii. Studies report the primary outcome with or without secondary outcomes.

c. Exclusion Criteria: The specific criteria for excluding studies are:

i. Observational studies, reviews, comments and letters to editors.

ii. Studies that do not report primary outcome.

iii. Grey literature.

iv. Duplicate studies.

v. Studies not retrievable in the English Language.

\section{d. PICOs}

Population: Individuals including oncology patients who are neutropenic and, or prophylactic use for peripheral stem cell mobilization in transplant donors.

Intervention: G-CSF biosimilars.

Comparator: Reference product, filgrastim.

Outcomes: The primary outcomes are effectiveness, uses and safety of G-CSF biosimilars. The secondary outcomes include overall improvement in cancer patients' quality of life, rationale for short vs long acting biosimilars and types of biosimilars approved and available in the market.

\section{Information Sources}


Our search uses sensitive topic-based strategies designed for each database. Nine databases will be searched: PubMed, CINAHL, Embase, Web of Science, Google Scholar, AJOL, Cochrane library, ResearchGate and Scopus will be included. Only randomized clinical trials and quasi-clinical trials, retrievable in the English langauges will be included.

\section{Search strategy}

The search strategy will include MeSH terms, text words, and entry terms. The search strategies used in databases are shown in Table 1.

\section{Data Extraction and Management}

\section{a. Data Extraction}

Data will be managed in three main softwares: DistillerSR, CMA version 3 and Microsoft Excel.

Identified studies will be screened independently in pairs and blindly using the DistillerSR software at 6 different levels:

i. Level 1 would involve screening of identified studies for the study design. Only interventional studies would be accepted

ii. Level 2 will involve screening of identified studies in the titles and abstracts using the predefined study characteristics

iii. Level 3 will involve further screening of the contents of articles by reading the full text articles using the search strategy.

iv. Level 4 will involve snowballing of literature on references from included studies.

v. Level 5: Studies will be screened for outcome measures of effectiveness, uses and safety of G-CSF biosimilars.

vi. Level 6: studies will be assessed for risk of bias using NIH Quality Assessment for Interventional studies and Cochrane Risk of Bias for Randomized Clinical trials.

Conflicts during screening will be resolved by a third independent reviewer who serves as a tie breaker.

\section{a. Selection Process:}

Screened studies will be selected based on study characteristics: study design, inclusion/exclusion criteria and agreement between two independent and blinded reviewers. Authors of included studies with missing data will be contacted via email and telephone. After selection, studies will be deduplicated. Data items will be extracted from selected studies into predefined forms in the DistillerSR.

b. Data Collection: Data items/measurable outcomes to be extracted from selected studies include:

i. Surname of first author and year of publication.

ii. Effectiveness measures: a) proportion of patients with 50\% increase in absolute neutrophil count within 3-7 days; b) proportion of fever resolved within 37 days, c) resolution of intra-oral mucosa ulcers within 7-10 days and d) resolution of difficulty in swallowing within 7-10 days.

iii. Measures of safety: a) proportion of patients with immunologic reactions, b) proportion of patients with any other documented adverse events.

iv. Uses of G-CSF biosimilars.

v. Secondary outcomes: a) improvement in cancer patients' quality of life, b) rationale for choice of short vs long acting biosimilars and c) types of biosimilars approved and available in the market.

Data items will be exported into predefined format in Microsoft Excel, to be imported into the CMA software for quantitative analysis.

The effect size for the primary outcome is prevalence. The effect sizes for secondary outcomes are some categorical and others quantitative.

\section{Risk of bias}

The risk of bias in the selected studies will be assessed for each study using the NIH Quality assessment tool for interventional studies. The NIH Quality assessment tool has 14 questions, with scores about 8 indicating good quality study. This will be cross-checked with the Cochrane tool of risk of bias assessment for randomized clinical trials. Publication bias in the selection of studies will be visually assessed using the funnel plot (trim and fill method) and test for asymmetry. Other statistical tests such as Egger's regression intercept, Begg and Mazumdar's rank correlation and Orwin's fail-safe $\mathrm{N}$ will be used where appropriate. Studies with extreme bias will be subjected to sensitivity testing using the include/exclude function in the CMA Software.

\section{Assessment of Meta-bias}

Meta-bias will be assessed as follows:

i. Method of testing/reporting of effectiveness and safety measures for biosimilars. This will be done at the outcome level.

ii. Index of reporting measurable outcomes in included studies: Studies that were reported in different indices but similar in outcome and design will be converted to the primary effect size, prevalence, based on individual case evaluation.

iii. Heterogeneity will be assessed at the study level using the Q statistics, and its $p$-value, $I^{2}, \bigotimes^{2}$ (Tau squared). As a rule of thumb, $I^{2}$ values of less than $40 \%$ will be considered low heterogeneity while values $>40$ but $<75 \%$ will be considered moderate and values $>75 \%$ are high. This is done at the study level.

\section{Data synthesis}




\section{Criteria for Data Synthesis}

Extracted data items, that reported primary outcomes with or without secondary outcomes will be used for both narrative synthesis and quantitative analysis.

The following criteria will be applied for analysis:

a. Studies that passed the methodological quality assessment using the NIH quality assessment tool, crosschecked with the Cochrane Risk of Bias tool will be included. The results will be presented in tabular format, indicating all the extractable data items and each study quality score.

b. All studies with primary outcomes will be used for narrative synthesis.

c. All studies with primary outcomes and secondary outcomes that pass heterogeneity tests and have good quality scores will be used for quantitative synthesis.

d. Further Analysis: Subgroup analysis will be performed using variables such as type of biosimilars and rationale for choice of short vs long acting biosimilars as moderators. Meta-regression will be performed on quantitative variables such patient's quality of life, proportion of patients with $50 \%$ increase in absolute neutrophil count with 3-7 days (dependent variable) and other quantitative measures of effectiveness.

e. Where heterogeneity exists, sensitivity testing using include/exclude functions in the CMA software will be performed.

f. Both fixed and random computational models will be used to compare pooled/ summary prevalence, standard error, variance and $95 \% \mathrm{Cl}$.

\section{Presentation and Reporting of Results:}

The pooled prevalence, standard error, variance, $95 \% \mathrm{Cl}$ of effectiveness, uses and safety of G-CSF biosimilars will be reported in forest plots. The relative weights assigned to studies will be reported in forest plots as well. Both NIH quality score and the Cochraine risk of bias will be presented in tables along with extracted measurable outcomes. Tests of heterogeneity will be included in the forest plots. Subgroup analysis will also be reported in forest plots. Metaregression and publication bias analysis will be presented in plots while results of sensitivity testing will be reported in a table.

\section{Discussion}

G-CSF biosimilars will be compared with the reference product, neupogen (filgrastin). The uses, effectiveness and safety of the biosimilars will be discussed. The study will also examine short and long acting biosimilars and compare their overall effectiveness.

GRADE: The strength of evidence from this study will be assessed using the NIH Quality assessment for systematic review and meta-analysis.

\section{List Of Abbreviations}

Cl: Confidence Interval

G-CSF: Granulocyte Colony Stimulating Factor

GRADE: Grades of Recommendation, Assessment, Development and

Evaluation.

PRISMA-P: Preferred Reporting Items for Systematic reviews and Meta-analysis Protocol

NIH: National Institute of Health

CMA: Comprehensive Meta-Analysis Software

PROSPERO: International Prospective Register for Systematic Reviews

\section{Declarations}

\section{Ethics and Dissemination}

Ethical approval will not be required, since this study will rely solely on secondary source of data. The study will be published in a peer-reviewed scientific journal.

\section{Authors Contributions}

EN conceived the project, EN, AD, CU, HO, SN, AN, RI, CE, JD, UA and CO designed the study. AD, CU and HO did PubMed searches; SN, AN and RI did AJOL and Embase searches; CE and JD did Google scholar searches; UA and CO did searches for CINAHL, Cochrane Database and Researchgate. AD, CU and HO used DistillerSR for screening at all levels with $\mathrm{HO}$ as a tiebreaker. SN and AN did NIH Quality score while RI and CE did Cochrane Risk of Bias assessment. EN and AD did CMA analysis, while UA, JD and CO did the NIH Quality assessment for systematic review and meta-analysis. CU did early draft of protocol while EN expanded the protocol fully. All authors reviewed, corrected and accepted the manuscript for submission.

\section{Funding}


The study is funded by the Molecular Pathology Institute, Enugu Nigeria.

\section{Support}

The Molecular Pathology Institute provided the DistillerSR via the Institute subscription.

Guarantor of the Review: Dr Emmanuel Nna

\section{Ethical approval and consent to participate}

Not applicable

\section{Consent for publication}

Not applicable

\section{Competing interest}

The authors declare no competing interest

\section{Acknowledgements}

We thank the staff of The Molecular Pathology Institute for helping with the training on DistillerSR.

\section{Amendments}

Important protocol amendments post registration will be recorded and included in dissemination.

\section{References}

1. American Society of Clinical Oncology. Recommendations for the use of hematopoietic colony-stimulating factors: evidence-based, clinical practice guidelines. J Clin Oncol. 1994;12(11):2471-2508. doi:10.1200/JCO.1994.12.11.2471

2. Dale DC. Colony-stimulating factors for the management of neutropenia in cancer patients. Drugs. 2002;62 Suppl 1:1-15. doi:10.2165/00003495200262001-00001

3. Anderlini P. Effects and safety of granulocyte colony-stimulating factor in healthy volunteers. Curr Opin Hematol. 2009;16(1):35-40. doi:10.1097/MOH.0b013e328319913c

4. Smith TJ, Khatcheressian J, Lyman GH, et al. 2006 update of recommendations for the use of white blood cell growth factors: an evidence-based clinical practice guideline. J Clin Oncol. 2006;24(19):3187-3205. doi:10.1200/JC0.2006.06.4451

5. Aapro MS, Bohlius J, Cameron DA, et al. 2010 update of EORTC guidelines for the use of granulocyte-colony stimulating factor to reduce the incidence of chemotherapy-induced febrile neutropenia in adult patients with lymphoproliferative disorders and solid tumours. Eur J Cancer. 2011;47(1):8-32. doi: 10.1016/j.ejca.2010.10.013

6. Bao-Guo X, Chuan-Zhen L, Hans L. J Cell Mol Med. 2007 Nov; 11(6): 1272-1290. Published online 2007 Aug 13. doi: 10.1111/j.1582-4934.2007. 00101.X PMCID: PMC4401293

7. Athanasia D. Panopoulos, Stephanie S. Watowich, Granulocyte colony-stimulating factor: Molecular mechanisms of action during steady state and 'emergency' hematopoiesis, Cytokine, Volume 42, Issue 3, 2008, Pp 277-288, ISSN 1043-4666, https://doi.org/10.1016/j.cyto.2008.03.002.

8. Dale DC, The discovery, development and clinical applications of granulocyte colony-stimulating factor. Trans Am Clin Climatol Assoc. 1998; 109: 27-38. PMCID: PMC2194342

9. Choi C, Yoo BW, Kim CO, et al. Comparison of biosimilar filgrastim with a reference product: pharmacokinetics, pharmacodynamics, and safety profiles in healthy volunteers. Drug Des Devel Ther. 2018; 12:2381-2387. Published 2018 Aug 1. doi:10.2147/DDDT.S158277

10. Caselli D, Cesaro S, Aricò M. Biosimilars in the management of neutropenia: focus on filgrastim. Biologics. 2016; 10:17-22. https://doi.org/10.2147/BTT.S73580

11. Corey SJ, Burkhardt AL, Bolen JB, Geahlen RL, Tkatch LS, Tweardy DJ. Granulocyte colony-stimulating factor receptor signaling involves the formation of a three-component complex with Lyn and Syk protein-tyrosine kinases. Proc Natl Acad Sci U S A. 1994;91(11):4683-4687. doi:10.1073/pnas.91.11.4683

12. Kawalec P, Stawowczyk E, Tesar T, et al. Pricing and reimbursement of biosimilars in Central and Eastern European countries. Front Pharmacol. 2017; 8:288. doi: 10.3389/fphar.2017.00288

13. AJMC 2017. Pricing and Contracting in Granulocyte Colony Stimulating Factors and Biosimilars for Febrile Neutropenia. Supplements and Featured Publications, Clinical Advance in the Management of Febrile Neutropenia. Accessed January 09, 2021, Available at https://www.ajmc.com/view/pricingand-contracting-in-granulocyte-colony-stimulating-factors-and-biosimilars-for-febrile-neutropenia

14. Siegel JF, Royzman I. Update on biosimilar approvals and pending applications in Europe and the U.S. Biologics Blog website. www.biologicsblog.com/update-on-biosimilar-approvals-and-pending-applications-in-europe-and-the-u-s. Published May 19, 2017.

15. Mulcahy AW, Predmore Z, Mattke S; Rand Corporation. The cost savings potential of biosimilar drugs in the United States. Rand Corporation website. www.rand.org/pubs/perspectives/PE127.readonline.html. Published 2014. 
16. Ventola CL. Biosimilars: part 1: proposed regulatory criteria for FDA approval. P T. 2013;38(5):270-287

17. Pahnke S, Egeland T, Halter J, et al. Current use of biosimilar G-CSF for haematopoietic stem cell mobilisation. Bone Marrow Transplant. 2019;54(6):858866. doi:10.1038/s41409-018-0350-y

18. European Medicines Agency 2019. Biosimilars in the EU (Informationguide for healthcare professionals).http://www.ema.europa.eu/docs/en_GB/document_library/Leaflet/2017/05/WC500226648.pdf.

19. European Medicines Agency 2019. Biosimilar medicinal productscontaining recombinant granulocyte-colony stimulating factor. http://www.ema.europa.eu/ema/index.jsp?curl=pages/regulation/general/general_content_001386.jsp\&mid=WC0b01ac058002958c.

20. European Medicines Agency 2019. Similar biological medicinal pro-ducts containing biotechnology-derived proteins as active sub-stance: non-clinical and clinicalissues.http://www.ema.europa.eu/ema/index.jsp?curl=pages/regulation/general/general_content_001386.jsp\&mid=WC0b01ac058002958c.

21. Pahnke S, Egeland T, Halter J, et al. Current use of biosimilar G-CSF for haematopoietic stem cell mobilisation. Bone Marrow Transplant. 2019;54(6):858866. doi:10.1038/s41409-018-0350-y

22. Caselli D, Cesaro S, Arico M. Biosimilars in the management of neutropenia: Focus on filgrastim. Biologics. 2016,10, 17-22.

23. Cancer Network 2002. Neulasta (pegfilgrastim) gets FDA approval to prevent infections.www.cancernetwork.com/articles/neulasta-pegfilgrastim-getsfda-approval-prevent-infections. Accessed, $27^{\text {th }}$ Jan 2021

24. OPM 2019. Udenyca (Pegfilgrastim-cbqv) Second Biosimilar Approved to Reduce the Incidence of Infection Associated with Febrile Neutropeniahttp://oncpracticemanagement.com/special-issues/2019-fourth-annual-oncology-guide-to-new-fda-approvals/1243-udenyca-pegfilgrastimcbqv-second-biosimilar-approved-to-reduce-the-incidence-of-infection-associated-with-febrileneutropenia\#: :text=The\%20originator\%20drug\%20pegfilgrastim\%20(Neulasta,acting\%20form\%20of\%20G\%2DCSF.\&text=In\%20June\%202018\%2C\%20th

25. Oncology Nursing News 2020. FDA Approves Pegfilgrastim Biosimilar. https://www.oncnursingnews.com/web-exclusives/fda-approves-pegfilgrastimbiosimilar. Accessed, $27^{\text {th }}$ Jan 2021.

26. Gascon P. The evolving role of biosimilars in haematology-oncology: a practical perspective. Ther Adv Hematol. 2015;6(6):267-281. doi: $10.1177 / 2040620715613715$

27. FDA 2018. Generic Drugs: Questions \& Answers, https://www.fda.gov/drugs/questions-answers/generic-drugs-questions-answers. Accessed, $27^{\text {th }}$ 2021

\section{Table}

Table 1: Search strategy in PubMed

(( (((GCSF) OR (GCSF Biosimilar)) OR (Granulocyte[Title] AND Colony[Title] AND Stimulating[Title] AND Factor[Title] AND Biosimilars[Title])) OR (((G-CSF OR Granulocyte Colony-Stimulating Factor OR Colony-Stimulating Factors OR) AND "Granulocyte Colony-Stimulating Factor"[Mesh] AND "biosimilar pharmaceuticals"[MeSH Terms] OR Biosimilars[Text Word] OR Biosimilar OR

Subsequent Entry Biologics) AND "Biosimilar Pharmaceuticals"[Mesh])) AND ((Neoplasia OR Neoplasias OR Neoplasm OR Tumors OR Tumor OR Cancer OR Cancers OR Malignancy OR Malignancies OR Malignant Neoplasms OR Malignant Neoplasm OR Benign

Neoplasms OR Benign Neoplasm) AND "Neoplasms"[Mesh])) AND ((Patient Relevant Outcome OR Clinical Efficacy OR Clinical Effectiveness OR Treatment Effectiveness OR Treatment Efficacy) AND "Treatment Outcome"[Mesh]) Filters: Clinical Trial, Randomized Controlled Trial

\section{Supplementary Files}

This is a list of supplementary files associated with this preprint. Click to download.

- PRISMAPchecklistforGCSFstudy.docx 